\title{
Third Order Optical Nonlinearity of Silver Nanoparticles Prepared by Chemical Reduction Method
}

\author{
Hussein T. Salloom \\ Al-Nahrain Nanorenewable Energy Research Center, Al-Nahrain University, Baghdad-Iraq. \\ Corresponding Author: abnthamer@gmail.com.
}

\begin{abstract}
In this study, silver colloidal nanoparticles have been prepared by chemical reduction of silver nitrate by trisodium citrate as a reduction agent. The adding time of the reduction agent was held constant while the time of stirring was varied. The resulting silver nanoparticles were characterized by UV-VIS absorption spectroscopy. The non-linear refractive index and absorption coefficient of silver nanoparticles were investigated using a single beam z-scan technique. The excitation source was a continuous wave $(\mathrm{CW})$ of $532 \mathrm{~nm}$ diode laser with a beam power of $40 \mathrm{~mW}$. All investigated samples showed negative and large thermally-induced non-linear refractive indices. Other nonlinear optical parameters (i. e. $\beta, \chi_{r}^{(3)}, \chi_{i}^{(3)}$ ) were also calculated. Our measurements confirm that the nonlinear parameters are caused by self-defocusing process making them potential candidates for nonlinear optical devices. [DOI: $\underline{10.22401 / J N U S .20 .3 .15]}$
\end{abstract}

Keywords: Silver colloidal nanoparticles, chemical reduction, nonlinear optical parameters, Z- scan.

\section{Introduction}

The nanomaterials with dimension less than $100 \mathrm{~nm}$ have received great attention from the scientific community not only for their unique properties but also for their versatile technological applications $[1,2]$. This interest stems from the distinctive change in electronic, magnetic, photonic, mechanical and catalytic properties as the particle size is reduced to nanoscale $[3,4]$. In this regard, the nonlinear response of the nanoparticles (NPs) to the interaction of light is dependent on their size and shape which are mainly related to the surface plasmons when the percentage of surface atoms in (NPs) is huge compared to that in the bulk counterparts $[5,6]$. It is worth highlighting that controlling the size and size distribution can be made by varying the synthesis methods and precursor materials. Silver (Ag) NPs have been produced using different methods including electrochemical method [7,8], thermal decomposition [9], laser ablation [10], microwave irradiation [11] and sonochemical synthesis [12]. The simplest and the most commonly used method for synthesis metal NPs is the chemical reduction of metal salts $[13,14]$. Thermally induced changes of refractive index caused by absorption of light in colloidal systems that consist of NPs dispersed in a liquid media have been intensively investigated both experimentally and theoretically [6]. Shahriari and Yunus investigated the thermal-induced non-linearity of $\mathrm{Ag}$ nano-fluid prepared by $\gamma$-radiation method using a single beam z-scan technique [15]. The sign of the non-linear refractive index, $\mathrm{n}_{2}$ was found to be negative and the maganitude was in the order of $10^{-8} \mathrm{~cm}^{2} / \mathrm{W}$. The results were attributed to the formation of thermal lens in the medium around metal NPs due to the effective heat transfer from the NPs to the medium [15]. Majles Ara et al. measured nonlinear refractive indices of $\mathrm{Ag}$ and $\mathrm{Au}$ particles using the Moire deflectometry with a $\mathrm{CW}$ of $\mathrm{He}-\mathrm{Ne}$ laser at wavelength of $632.8 \mathrm{~nm}$. The nonlinear refractive induces for $\mathrm{Ag}$ and $\mathrm{Au}$ NPs obtained at $50 \mathrm{~mW}$ were found equal to $1.8 \times 10^{-7}$ and $1 \times 10^{-8} \mathrm{~cm}^{2} / \mathrm{W}$ respectively [16]. In this work, we report on the characterization of Ag NPs synthesized by chemical reduction method with different stirring durations after the addition of a reducing agent. Z-scan technique, which has emerged as a powerful method to determine the non-linear refraction of colloidal metal NPs, has been employed to study the non-linear refractive response from Ag NPs.

\section{Materials and Methods}

Silver nitrate $\left(\mathrm{AgNO}_{3}\right)$ was used as starting material while trisodium citrate $\left(\mathrm{C}_{6} \mathrm{H}_{5} \mathrm{O}_{7} \mathrm{Na}_{3}\right)$ was used as reducing agent and the solution of 
reaction was prepared in distilled water. In this research $50 \mathrm{ml}$ of $1.06 \mathrm{mM}$ of aqueous silver nitrate was heated up to boiling temperature, to this solution $10 \mathrm{ml}$ of $38 \mathrm{mM}$ of trisodium citrate solution was added drop wise to the $\mathrm{AgNO}_{3}$ solution. The addition of $10 \mathrm{ml}$ of trisodium citrate lasted 4 minutes during which stirring and boiling of the solution were maintained. The specimens of the resulting solution were collected at three durations of 10, 25 and 40 minutes after adding trisodium citrate. These specimens are labeled as $S_{1}, S_{2}$ and $S_{3}$. The changing in solution color to yellow indicates the formation of Ag NPs.

The mechanism of reaction could be given by the following chemical equation [17]

$4 \mathrm{Ag}^{+}+\mathrm{C}_{6} \mathrm{H}_{5} \mathrm{O}_{7} \mathrm{Na}_{3}+2 \mathrm{H}_{2} \mathrm{O} \rightarrow 4 \mathrm{Ag}^{0}+\mathrm{C}_{6} \mathrm{H}_{5} \mathrm{O}_{7} \mathrm{H}_{3}$ $+3 \mathrm{Na}^{+}+\mathrm{H}^{+}+\mathrm{O}_{2} \uparrow$

The linear absorption spectra were measured by a double beam UV-VIS spectrophotometer SPUV-26 in the spectral range between 190 and $1100 \mathrm{~nm}$ using a quartz cell having a thickness of $10 \mathrm{~mm}$. The Z-scan experimental setup was analogous to that described in Reference [18]. The measurements performed using a continuous wave (CW) laser diode delivering at wavelength of $532 \mathrm{~nm}$ and out power of $40 \mathrm{~mW}$. The laser beam was focused to a small spot by a convex lens of $100 \mathrm{~mm}$ focal length to a waist radius $\left(\omega_{0}\right)$ of $33.8 \mu \mathrm{m}$, the diffraction length $Z_{0}=\frac{\pi \omega_{0}^{2}}{\lambda}$ of $6.7 \mathrm{~mm}$. The on-axis power density of the laser beam at the waist $I_{0}$ was calculated to be $2.2 \mathrm{~kW} / \mathrm{cm}^{2}$. The sample was infiltrated in $2 \mathrm{~mm}$ thick quartz cell, the cell was hold on homemade translational stage to move freely across the focal region along the $\mathrm{z}$ - axis, aperture of $0.2 \mathrm{~mm}$ was placed before the power meter. The required condition $\left(\frac{\pi \omega_{0}^{2}}{\lambda}\right)>\mathrm{L}$ is satisfied, where $\omega_{0}$ is beam waist at the focal point, $L$ is the sample thickness and $\lambda$ is the wavelength of the laser light.

\section{Results and Discussion}

During the synthesis of silver colloidal nanoparticles, the addition time of trisodium citrate was constant but the time of stirring after the addition of reducing agent was varied. The UV-VIS absorption spectrum of Ag nano-colloids is shown in Fig.(2). There is a strong absorption in the visible region. This unique optical behavior is due to surface plasmons resonance which is the collective oscillations of free electrons at metaldielectric interface and electromagnetic fields associated with these oscillations [19]. The absorption peaks above $400 \mathrm{~nm}$ indicate the formation of $\mathrm{Ag}$ nanoparticles [20]. The increase in maximum absorbance at different stirring time is due to increase in the amount of silver nanoparticles. On the other hand, a slight red shift in the position of absorption peaks indicates that the particles aggregate to larger size by increasing the stirring time [21]. For all samples, the mixture undergoes a color change from transparent to a yellow color as primary blue was absorbed. The color of nano particles was found to be highly depended on their preparation, with larger particles and more asymmetric particles absorbing at longer wavelengths than smaller and more spherical particles [22].

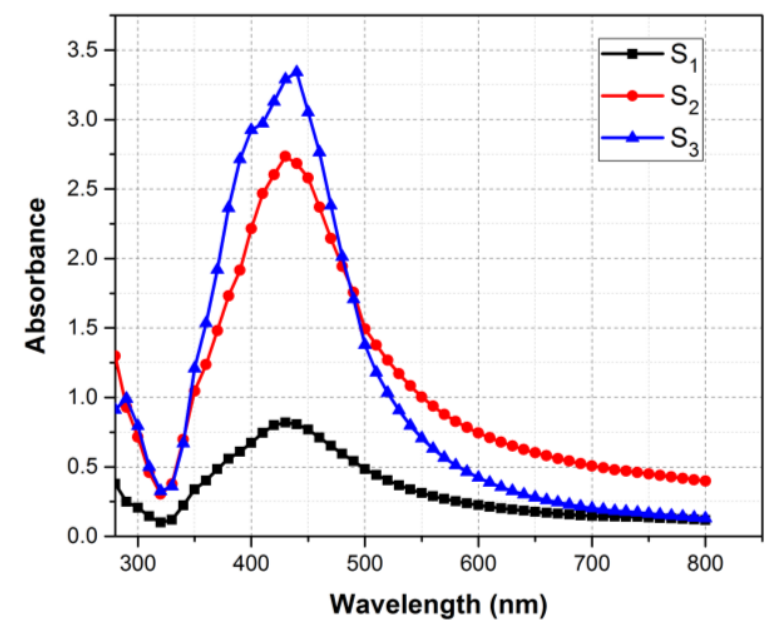

Fig.(2): Absorption spectra of colloidal silver nanoparticle solutions collected at different stirring times. $\left(S_{1}\right.$ collected at $10 \mathrm{~min}, S_{2}$ collected at 25 min and $S_{3}$ collected at 40 min).

The nonlinear optical characteristics of prepared nano-sized silver colloidal samples are investigated by performing Z-scan technique [23]. Z-scan measurements allow to determine both nonlinear refractive index $\mathrm{n}_{2}$ and nonlinear absorption coefficient $\beta$ using closed and open aperture procedures as described by Sheik Bahaa et al. [24].

The normalized transmission curves obtained using closed aperture set up for $\mathrm{Ag}$ 
nanoparticles samples are shown in Fig.(3). The measurement of the distance between the peak and valley starts far away from the focus (negative $\mathrm{z}$ ), where the transmittance is relatively constant. Then the sample is moved towards the focus and then to the positive $\mathrm{z}$. The nonlinear refractive index of $\mathrm{Ag}$ nano colloidal solution is estimated using the following equation [25]:

$n_{2}=\frac{\Delta \varphi_{0} \lambda}{2 \pi I_{0} L_{e f f}}$

where $\Delta \varphi_{0}$ is the on- axis phase shift at the focus. The most important quantity could be obtained by the best theoretical fit from the results is $\Delta T_{p-v}$, which represent the dierence between the highest (peak) and the lowest (valley) value of transmittance i.e. $T_{p}-T_{v}$. The relation between $\Delta T_{p-v}$ and $\Delta \varphi_{0}$ which is a function of $\mathrm{n}_{2}$ is given as:

$\Delta T_{p-v}=0.406(1-S)^{0.25}\left|\Delta \varphi_{0}\right|$

Therefore, $\mathrm{n}_{2}$ can be evaluated by using:

$n_{2}=\frac{\Delta T_{p-\nu} \lambda}{0.406(1-S)^{0.25} 2 \pi I_{0} L_{e f f}}$

where $S$ is the linear transmittance at aperture $(S=0.39)$ for the present experimental set up, $I_{0}$ is the laser beam intensity at focus, $L_{\text {eff }}=\left(1-\exp -\alpha_{0} L\right) / \alpha_{0}$ is the effective thickness of the specimen, $\alpha_{0}=-\frac{1}{L} \operatorname{Ln}\left(\frac{I}{I_{0}}\right)$ is the linear absorption coefficient and $\mathrm{L}$ is thickness of the sample. In Fig.(3) square symbols represent the experimental data while the solid lines are the fitting of normalized transmittance data to the closed aperture formula [18]

$$
T(z)=1+\frac{4 \Delta \varphi_{0} x}{\left(x^{2}+1\right)\left(x^{2}+9\right)}
$$
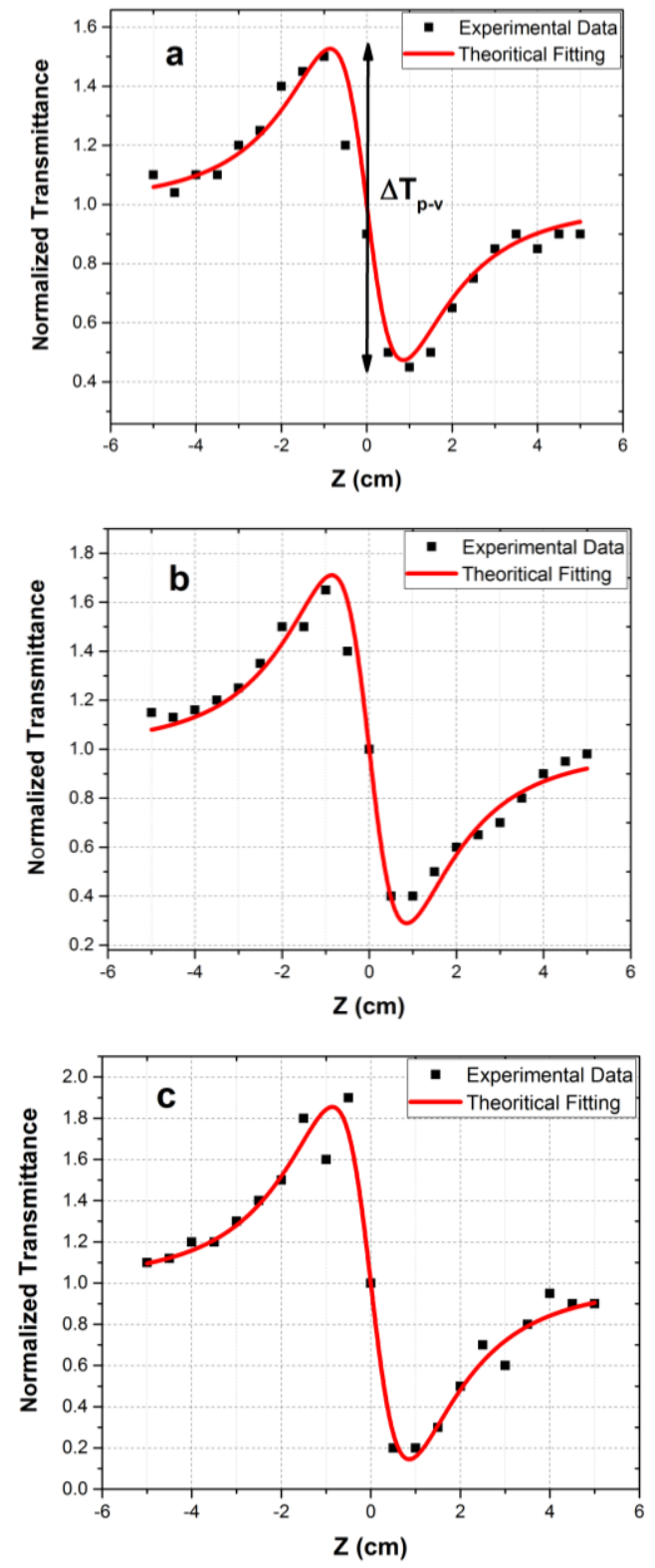

Fig.(3): Closed aperture transmittance curves of synthesized silver nanoparticle samples.

(a) for sample $S_{1}$, (b) for sample $S_{2}$ and (c) for sample $S_{3}$.

The nonlinear refractive index $\mathrm{n}_{2}$ can be determined from the normalized transmittance data of the closed aperture measurements. Normalized peak and valley transmittance $\Delta T_{p-v}$ was calculated to be $-1.2,-1.45$ and -1.7 for sample $S_{1}, S_{2}$ and $S_{3}$ respectively. Then the values of nonlinear refractive indices are found as summarized in Table (1). The values reported in the present work were in the same order with that reported in related researches [26]. The nonlinear refractive index sign is negative and clearly indicate the thermal selfdefocusing phenomena. The values of $\mathrm{n}_{2}$ increase with the increase of post addition 
stirring and this due to increase of thermo optic effect as the $\mathrm{Ag}$ nano particles aggregating to larger particles with increasing stirring time. In addition, the increasing in $\mathrm{n}_{2}$ can be due to aggregation effects of nanoparticles when the concentration of silver solution increase. The nonlinear refractive indices are calculated as $\left(-8.98 * 10^{-8},-1.11 * 10^{-7}\right.$ and $\left.-1.33 * 10^{-7}\right) \mathrm{cm}^{2} / \mathrm{W}$.

The normalized transmission curves obtained using open aperture set up for $\mathrm{Ag}$ nanoparticles samples is shown in Fig.4. The square symbols represent the experimental data while the solid lines are the curve fit to the analytical equation. Nonlinear absorption coefficient $\beta$ in $(\mathrm{cm} / \mathrm{W})$ can be measured by fitting normalized transmittance data into the formula given by $[23,25]$.

$$
T(z, s=1)=1-\left[\frac{I_{0} \beta L_{e f f}}{2^{\frac{3}{2}}\left(1+\frac{z^{2}}{z_{0}^{2}}\right)}\right]
$$

and

$$
\beta=\frac{2 \sqrt{2} \Delta T}{I_{0} L_{e f f}}
$$

where $\Delta \mathrm{T}$ is the one-valley value at the open aperture Z-scan fitting curve and $\beta$ is nonlinear absorption coefficients (the fitting parameter). An open aperture normalized transmission of all samples is measured in the far field region. As can be clearly noticed in Fig.(4), a decrease in values of transmission around the focal region $(\mathrm{z}=0)$ indicate the nonlinear absorption in silver nano colloidal. This behavior could be attributed to the two photon absorption and excited state absorption phenomena [26]. It has been noticed that the values of $\beta$ decrease with the increase of post adding stirring time that caused to the increase of the particle sizes. If the particle become larger a small number of nano particles will accommodate in the same volume i.e. decrease in fraction volume, and, consequently, $\beta$ decreased [26]. The nonlinear absorption coefficients for present samples are listed in Table (1). Researchers addressed that various physical mechanisms contribute independently at different time scales to optical nonlinearities of the material. Generally, the nonlinear refraction arises from the electronic effect or thermal mechanisms [27]. In the solution the thermal effect is a slow accumulative process which is associated with the non-radiative relaxation of the excitation energy gathered via linear or nonlinear absorption by the metal NPs. For the aqueous solution the buildup time of such thermal effect is about 30 ns [28]. Thermal response will be prominent using CW laser or long laser pulses since heat can accumulate and cause acoustic waves that change the density of the samples. The thermal nonlinearity of nanoparticles solution can be enhanced using solvents with thermo optic coefficient and low thermal conductivity such as ethanol [29].
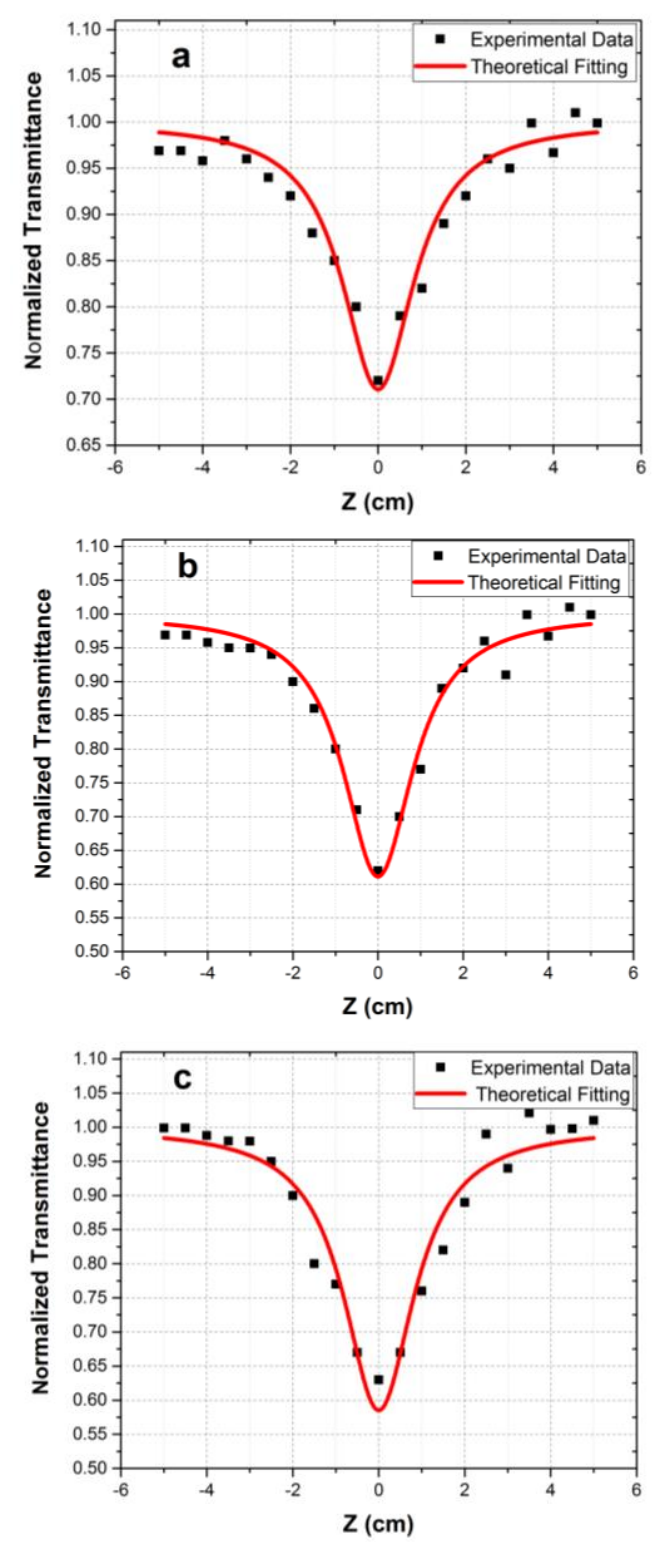

Fig.(3): Open aperture transmittance curves of synthesized Ag nano particles samples.

(a) for sample $S_{1}$, ()b for sample $S_{2}$ and (c) for sample $S_{3}$.

By knowing the nonlinear constants $\mathrm{n}_{2}$ and $\beta$ one could obtain the real $\chi_{r}^{(3)}$ and imaginary 
$\chi_{i}^{(3)}$ parts of third order susceptibility $\chi^{(3)}$ using the following equations [25]:

$$
\begin{aligned}
& \chi^{(3)}=\chi_{r}^{(3)}+i \chi_{i}^{(3)} \\
& \operatorname{Re} \chi^{(3)}(e s u)=10^{-4} \frac{\varepsilon_{0} c^{2} c}{\pi} n_{2} \\
& \operatorname{Im} \chi^{(3)}(e s u)=10^{-2} \frac{\varepsilon_{0} c^{2} n_{0}^{2} \lambda}{4 \pi^{2}} \beta
\end{aligned}
$$

The absolute value of third order is calculated by [22]:

$\left|\chi^{(3)}\right|=\left[\left(\operatorname{Re} \chi^{(3)}\right)^{2}+\left(\operatorname{Im} \chi^{(3)}\right)^{2}\right]^{1 / 2}$

where $\mathrm{n}_{0}$ is the linear refractive index of refraction, $\mathrm{c}$ is the speed of light, and $\varepsilon_{0}$ is the free space permeability. All the results are listed in Table (1).

Table (1)

Calculated Values of nonlinear parameters for prepared Ag nano particles colloidal.

\begin{tabular}{|c||c||c||c||c||c||c||c||}
\hline Ag samples & Stirring time & $\Delta \varphi_{0}$ & $n_{2}\left(\mathrm{~cm}^{2} / W\right)$ & $\operatorname{Re} \chi^{(3)}$ & $\beta(\mathrm{cm} / W)$ & $\operatorname{Im} \chi^{(3)}$ & $\left|\chi^{(3}\right|$ \\
\hline \hline S1 & 10 & -1.2 & $-8.90 \mathrm{E}-8$ & $-5.17 \mathrm{E}-6$ & $3.81 \mathrm{E}-3$ & $9.31 \mathrm{E}-5$ & $9.34 \mathrm{E}-5$ \\
\hline \hline S2 & 25 & -1.5 & $-1.11 \mathrm{E}-7$ & $-6.41 \mathrm{E}-6$ & $3.64 \mathrm{E}-3$ & $8.91 \mathrm{E}-5$ & $8.93 \mathrm{E}-5$ \\
\hline \hline S3 & 40 & -1.7 & $-1.33 \mathrm{E}-7$ & $-7.72 \mathrm{E}-6$ & $2.61 \mathrm{E}-3$ & $6.38 \mathrm{E}-5$ & $6.40 \mathrm{E}-5$ \\
\hline
\end{tabular}

\section{Conclusions}

In summary, silver nanoparticles are chemically reduced in trisodium citrate and collected at 10, 25 and $40 \mathrm{~min}$. post adding stirring time. The optical absorption of $\mathrm{Ag}$ nano colloidal have been investigated using UV-VIS spectroscopy. Surface plasma resonance band of silver nanoparticle is found at $428 \mathrm{~nm}, 430 \mathrm{~nm}$, and $440 \mathrm{~nm}$ and shows a small red shift. Nonlinear optical parameters are estimated using $\mathrm{z}$-scan technique. Nonlinear refractive index of silver nanoparticles is found to be negative and as high as $\left(-8.98 * 10^{-8},-1.11 * 10^{-7}\right.$ and $\left.-1.33 * 10^{-7}\right)$ $\mathrm{cm}^{2} / \mathrm{W}$. The variation of nonlinear coefficient of samples as stirring time increase were reported. High nonlinear refractive index is due to thermo-optic effect. The thermal induced optical effect could be used as a thermal lens for many photonic applications such as optical limiting and beam deflection.

\section{References}

[1] Basavaraj U., Praveenkumar N., Sabiha T.S., Rupali S., Samprita B., "Synthesis and Characterization of Silver Nanoparticles" Research Article Biological Sciences, IJPBS 2(3), 10-14, 2012.

[2] Sally D. S., Mozghan B., Aravindan V., Jeyarajasingam, Susan A. R. and Charles B. "Synthesis and Study of Silver Nanoparticles" J. Chem. Educ., 84 (2), p 322, 2007.
[3] Suriati G., Mariatti M. and Azizan A., "Synthesis Silver Nanoparticles by Chemical Reduction Method: Effect of Reducing Agent and Surfactant Concentration" International Journal of Automotive and Mechanical Engineering (IJAME) 10, 1920-1927, 2014.

[4] Ratyakshi and R.P. C. "Colloidal Synthesis of Silve r Nano Particles" Asian Journal of Chemistry, 21(10) 113-116, 2009.

[5] Gamernyk. R., Periv, M., Malynych. S., "Nonlinear Optical Refraction of Silver Nanoparticle Composites", Optica Applicata, Vol. XLIV, No. 3, 2014.

[6] Mashayekh M., and Dorranian D., "Size dependent nonlinear optical properties and thermal lens in silver nanoparticles," Optik,.125,5612-5618, 2014.

[7] Abou El-Nour K. M., Eftaiha A, AlWarthan A., Ammar RA., "Synthesis and applications of Silver Nanoparticles. Arabian Journal of Chemistry.;3, 135- 40, 2010

[8] Maribel G. G., Jean D., Stephan G., "Synthesis of silver nanoparticles by chemical reduction method and their antibacterial activity" International Journal of Chemical and Biomolecular Engineering 2,3 2009.

[9] Jung J.H., Cheol Oh. H., Soo Noh H., Ji JH, Soo Kim S. "Metal Nanoparticle generation using a Small Ceramic Heater with a Local Heating Area", Journal of Aerosol Science. 37, 1662-70, 2006. 
[10] Tarasenko N, Butsen A, Nevar E, Savastenko N. "Synthesis of Nanosized Particles during Laser Ablation of Gold in Water". Applied surface science, 252:4439-44, 2006.

[11] Taguchi A., Fujii S., Ichimura T., Verma P., Inouye Y., Kawata S.” Oxygen-assisted shape control in polyol synthesis of silver nanocrystals". Chemical Physics Letters. 462:92-5, 2008.

[12] Cai M., Chen J., Zhou J. "Reduction and morphology of silver nanoparticles via liquid-liquid method". Applied Surface Science, 226:422-6, 2004.

[13] Chou K-S., Ren C-Y. "Synthesis of Nanosized Silver Particles by Chemical Reduction Method", Materials Chemistry and Physics, 64:241-6, 2000.

[14] Hongshui W. O., Jianguo C., Shiyuan D., "Preparation of Silver Nanoparticles by chemical Reduction Method", Colloidal and Surfaces A: Physicochem. Eng. Aspects 256,111-115, 2005.

[15] Shahriari, E., Yunus,W.M., "Effect of Particle Size on Nonlinear Refraction and Absorption Ag Nanoparticles", Digest Journal of Nanomaterials and Biostructure. 5 (4), 939-946,2010.

[16] Majles ara., M.; Javadi, Z.; Sirohi, R.; "Measurement of nonlinear refractive index of $\mathrm{Ag}$ and $\mathrm{Au}$ nano-particles using moiré technique" Optik-International Journal for Light and Electron Optics 122(21):1961-1964; 2011.

[17] Ki Chang S., Sung Min L., Tae Sun P., and Bum Suk L., "Preparation of colloidal silver nanoparticles by chemical reduction method", Korean J. Chem. Eng., 26(1), 153-155, 2009.

[18] Zidan M.D. and Allahham A., "Z-Scan Measurements of the Third-Order Optical Nonlinearity of a C60 Doped Poly dimethylacetylendi-carboxylate", Acta Physica Polonica A, 7 (1), 128, 2015.

[19] Zaheer K., Shaeel A., Abdullah Y., A.O. Al-Youbi, "Preparation and characterization of silver nanoparticles by chemical reduction method", Colloids and Surfaces B: Biointerfaces 82, 513-517, 2011.

[20] Fang. Y. "Optical absorption of nanoscale colloidal silver: Aggregate band and adsorbate-silver surface band", Journal of Chemical Physics, 108(10):4315-4318, 1998.

[21] Craig F. B. and Donald R. H. "Absorption and scattering of light by small particles" John Wiley and Sons, Inc., Weinheim, Germany, 1983.

[22] Jack B. "Chemical Synthesis Of Silver Nanoparticles for Light Trapping Application in Silicon Solar Cell" Msc Thesis, University of Oslo, June 2010.

[23] Sheikbahae, M.; Said A. A.; Wei,T. H.; Hagan D. J. and Vanstryl and, E. W.; "Sensitive Measurement of Optical Nonlinearities Using a Single Beam," IEEE Journal of Quantum Electronics, 26 (4), 760-769, 1990.

[24] Sheikbahae, M., Said A. A.; Hagan; Soileau D. J., M. J. and Vanstryl and E. W., "Nonlinear Refraction and Optical Limiting in Thick Media", Optical Engineering, 30 (8), 1228-1235, 1991.

[25] Shahriaria E., Moradia, M., and Varnamkhastia, M., "Investigation of nonlinear optical properties of $\mathrm{Ag}$ nanoparticles", International Journal of Optics and Photonics (IJOP), 9(2), 107113, 2015.

[26] Mashayekh M. and Dorranian D., "Size dependent nonlinear optical properties and thermal lens in silver nanoparticles," Optik, vol. 125, 5612-5618, 2014.

[27] Faraji N., Yunus M. Kharazmi. A, Saion E., Shahmiri M., Tamchek, N., "Synthesis, characterization and nonlinear optical properties of silver/PVA nanocomposites". $\mathrm{J}$.

[28] Zhengle M., Lingling Q., Fei H., Yang L., Chen W., and Ya C, "Thermal-induced nonlinear optical characteristics of ethanol solution doped with silver nanoparticles", Chinese Optics Letters, 7,(10), 949, 2009.

[29] Alesenkov A., Pilipavičius J., Beganskienè A., Sirutkaitis R., and Sirutkaitis V.; Lithuanian Journal of Physics, Nonlinear Properties of Silver Nanoparticles explored by a Femtosecond Z-scan Technique'. 55(2), 100-109, 2015. 